\title{
Bridging the Gap: Utilizing Socratic Inquiry Facilitation in Cross- Cultural Communication and Conflict Exploration
}

\author{
Mikaela G. Zimmerman \\ West Virginia University, mgz0001@mix.wvu.edu
}

Follow this and additional works at: https://researchrepository.wvu.edu/etd

Part of the Civic and Community Engagement Commons

\section{Recommended Citation}

Zimmerman, Mikaela G., "Bridging the Gap: Utilizing Socratic Inquiry Facilitation in Cross-Cultural Communication and Conflict Exploration" (2019). Graduate Theses, Dissertations, and Problem Reports. 3908.

https://researchrepository.wvu.edu/etd/3908

This Thesis is protected by copyright and/or related rights. It has been brought to you by the The Research Repository @ WVU with permission from the rights-holder(s). You are free to use this Thesis in any way that is permitted by the copyright and related rights legislation that applies to your use. For other uses you must obtain permission from the rights-holder(s) directly, unless additional rights are indicated by a Creative Commons license in the record and/ or on the work itself. This Thesis has been accepted for inclusion in WVU Graduate Theses, Dissertations, and Problem Reports collection by an authorized administrator of The Research Repository @ WVU. For more information, please contact researchrepository@mail.wvu.edu. 
Bridging the Gap:

Utilizing Socratic Inquiry Facilitation in Cross-Cultural Communication and Conflict Exploration

Mikaela G. Zimmerman

Thesis submitted

to the Eberly College of Arts and Sciences

at West Virginia University

in partial fulfillment of the requirements for the degree of

Master of Arts in

Sociology

\author{
Jesse Wozniak, Ph.D., Chair \\ Joshua Woods, Ph.D. \\ James Nolan, Ph.D. \\ Department of Sociology and Anthropology
}

\title{
Morgantown, West Virginia \\ 2019
}

Keywords: dialogue, Socratic Inquiry, Socratic Method, facilitation, content analysis, gender Copyright 2019 Mikaela G Zimmerman 


\begin{abstract}
Bridging the Gap:

Utilizing Socratic Inquiry Facilitation in Cross-Cultural Communication and Conflict Exploration
\end{abstract}

\title{
Mikaela G Zimmerman
}

The objective of this research is to explore the possibilities for improving governmental relations (local, national, and international) using microcosms of cross-cultural dialogues facilitated with Socratic Inquiry methodology and their potential to project situational similarities into more formal political conversation. Using twenty-five recorded dialogues from The Pennsylvania State University's Center for Public Diplomacy (World in Conversation), I have broadly observed the role of Socratic Inquiry facilitation in cross-cultural dialogues whose content varies across a span of contentious social topics. By generally accounting for a range of relevant social factors such as gender, body language, conversational tools, and proxies for empathy, I am able to cultivate a broad picture of how people with differing cultural backgrounds connect with and find empathy for one another in settings of contentious social conversation with the help of facilitators trained in Socratic Inquiry methodology. I posit that these findings can be translated into higher-level political settings and serve as a flexible canvas for which fairer and more inclusive agreements can be reached. 


\section{TABLE OF CONTENTS}

INTRODUCTION

LITERATURE REVIEW: Microdialogue and Macropolitics ........................................................ 4

Gendered Individual and Relative Perceptions of Power .................................................................. 5

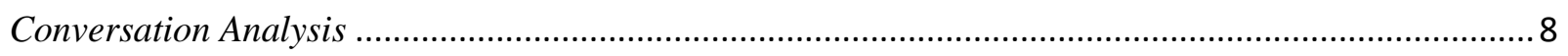

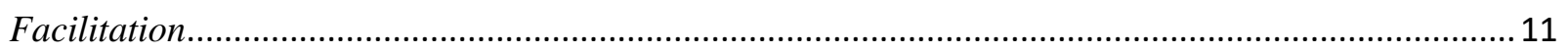

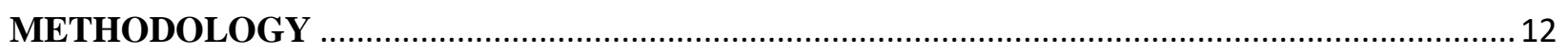

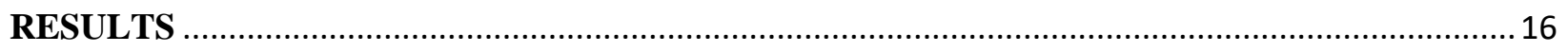

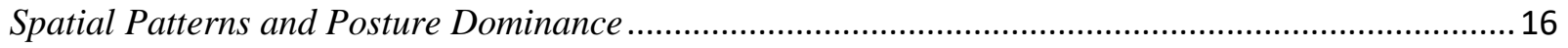

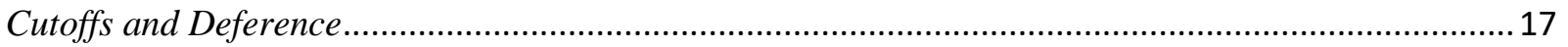

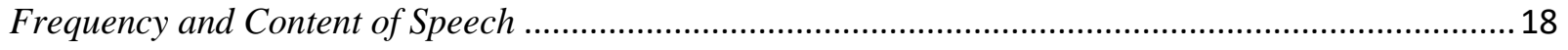

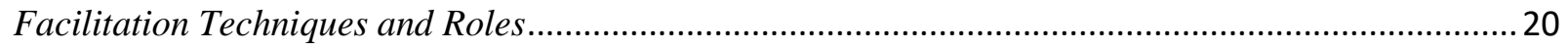

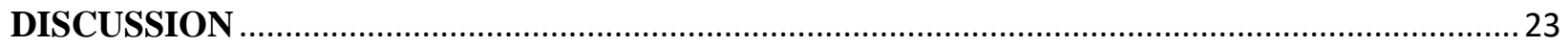

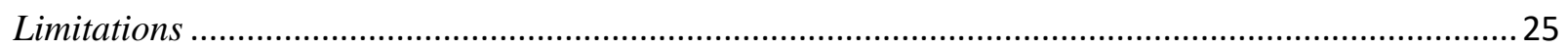

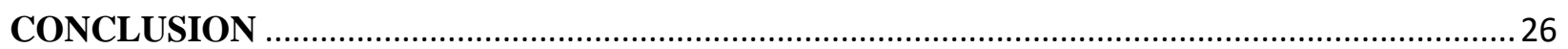

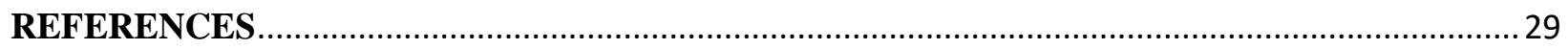


BRIDGING THE GAP 1

\section{INTRODUCTION}

Socratic Inquiry refers to a method of dialogic facilitation employing questions and reflections for purposes of exploration and critical thinking rather than objective education and agenda (Paul and Binker 1990); the goal is to allow participants to lead themselves and their peers to places of new or broadened understanding of each other's viewpoints. This methodology's open and exploratory nature lends itself to many spectrums of usage, among which is the world of governmental relations - one of unrivaled importance in a country with the power and influence of the United States. Due to the increasing diversity in the political scape, both in the United States and throughout the world, political conversations are beginning to encompass people of more and more diverse social backgrounds. As one can imagine, this realm of cross-cultural communication is brimming with cultural differences-religion, social customs, personal values, financial priorities, and much more - that effect how people connect with one another.

Part of the Socratic Inquiry methodological process is the cultivation of empathy among dialogue participants with aid from facilitators and certain facilitation techniques. A 2002 study found that empathy for someone else may increase the likelihood of fair treatment toward them (Page and Nowak 2002). They note that, although it is important to consider that some people define empathy as, "do-as-you-would-be-done-by" (Binmore 2001), their theory deals more with the evolution of fairness and therefore define empathy as, “...individuals make offers which they themselves would be prepared to accept" (Page and Nowak 2002). Additional findings are consistent with the view that empathic concern may instigate helping behavior (Lamm, Batson, and Decety 2007). Merging this idea with theories on empathy and fairness, I posit that facilitated interpersonal connection could be an important factor in cultivating empathy and 
fairness in cross-cultural communication, and therefore in settings of cross-cultural conflictincluding governmental relations.

One way to better understand this process is to observe how people with culturally diverse backgrounds connect with one another in dialogues facilitated with Socratic Inquiry methodology — how do we cultivate empathy, and what is the role of Socratic Inquiry facilitation in this cultivation process? What is important to talk about, and what kinds of dialogic tools and behaviors can we observe within this process of building connections between people? World in Conversation (WinC), the center for public diplomacy at The Pennsylvania State University, aims to shed light on the importance of perspective-sharing in the world of cross-cultural human connection. Utilizing twenty-five of WinC's archived "international dialogues" (dialogues between domestic American students and international students), I attempt to draw general microcosmic patterns of cross-cultural human connection within the context of dialogue content and gendered interaction/proxemics, as well as clarify the role of facilitation in conflict and perspective-sharing. These observations will act as a framework from which similar settings of conflict and contention can build and employ Socratic Inquiry methodology. Among presentation of findings, I will posit potential benefits of this facilitation in the world of cross-cultural communication in governmental relations.

The purpose of this study is to explore how people with varying cultural backgrounds form connections with one another in settings of open dialogue facilitated with Socratic Inquiry methodology (in this case, within the organization World in Conversation) and then use findings to make suggestions for better governmental—local, national, or international—relations. Synthesized pieces of cross-cultural dialogue such as gender interaction, conversational analysis, nonverbals/proxemics, and empathy cultivation will work together to reveal a malleable — and 
therefore applicable to a variety of like environments - microcosm of interaction, discussion, connection, and mediation of international and domestic (United States) participants. Broadly understanding how these factors may interact in other settings of contention and collaboration allows for informed application of findings to larger scale macrocosms such as governmental relations.

World in Conversation is a student-run center for public diplomacy whose mission is to connect people through exploring angles and perspectives of various contentious social topics with facilitators trained in Socratic Inquiry methodology (Center for Public Diplomacy). Every semester, certain professors at The Pennsylvania State University require their students to attend a dialogue at World in Conversation for class credit; the population of this research are those consenting adults participating in facilitated dialogues with topics specific to international relations with students from all around the world. The purpose of this is to observe how participants of different genders and backgrounds form cross-cultural connections with one another around contentious social topics. Observing differences between genders allows for a more accurate understanding of how people interact with one another in dialogue. Gender roles and norms certainly change throughout cultures, but a general understanding of gendered trends and patterns within different aspects of this research will help paint a clearer picture of how to best apply findings. These data can then be used to project theory and suggestions into the larger realms of international connection, diplomacy, and ambassadorship.

The issues inspiring this research stem from the grey areas of cross-cultural communication and the splintered state of United States international relations. The objective of this research is both exploratory and activist in nature, analyzing human connection within contexts of certain topics, proxemics, and socialized gender dynamics at work as well as the role 
of facilitation in cross-cultural dialogue. Observing purposefully facilitated dialogue will allow for a controlled environment in which participants can think critically and honestly. Gauging the effects of something as engrained in one's subconscious as gender dynamics and body language will help to provide small but important context for which to explore the role of facilitation in building cross-cultural communication in an environment illustrating a microcosm of everyday interactions. This microcosm of connection and mediation between Americans and international citizens will serve as an example which we can apply to the larger scope of national and international relations as well as more local legislative meetings where cultural diversity is present. This will serve to bring intention and clarity to the world of governmental relations by which we are all affected in some way, and to make suggestions for improving the current process of community and governmental diplomacy.

\section{LITERATURE REVIEW: Microdialogue and Macropolitics}

There are several layers to observe within a project such as this one, the first of which is gender. Gender permeates the entirety of this study because it is such an important layer in a project studying how people with different cultural backgrounds interact and connect with one another. Conversation analysis and understanding how participants are connecting and interacting with one another are also crucial to maximizing the applicability of this study. Elements of conversation analysis include: how participants are sharing information, what kind of information they are sharing (personal or otherwise), how the conversation advances and delays, and overall group dynamic (talkativeness, enthusiasm, etc.).

These smaller, more specific pieces of conversation analysis will serve as a theoretical base for which I can apply my observations of each dialogue, shedding light on how conversation changes and evolves between Americans and international citizens in settings of 
facilitated conflict and collaboration. This microcosm can then provide an idea of how these observations may compare to larger-scale interactions between people of varying cultural backgrounds in contentious settings, and even offer new, more effective means of cross-cultural communicational conduct.

\section{Gendered Individual and Relative Perceptions of Power}

Gender is important in this analysis because men and women behave differently according to their individual cultures. But even with this cultural element removed, men and women interact differently with each other than with members of their own gender (Smith-Lovin and Ridgeway 1999). When studying nuanced interaction between men and women, it is important to consider how men and women see each other in different settings. One way to do this is to observe men and women in dialogue with one another. By observing men and women in dialogue, we can use small-scale observations to infer large-scale impacts within society. These inferences can then be paired with conversation analysis and observation of various conversational techniques, specifically regarding connection between participants. By analyzing gender dynamics in interaction, conversational techniques in inter-participant connection, and the role of facilitation in dialogue, we can create potential new avenues for various mediums of cross-cultural communication, specifically within the political realm of diverse governmental relations.

To understand how men and women communicate in dialogue, we must explore the daily operations and definitions of men and women in society. It would be useful to look at these factors through lenses of gender socialization and cultural expectations of genders for purposes of interpreting interaction between men and women. For example, the role that perception of others' opinions plays when relating to the formation of public opinion is an important gauge of 
how interacting parties will view one another in relation to themselves. Put more simply, what do people think of other people's opinions? Men and women may experience relative selfassessment differently, therefore informing an aspect of how they connect with one another in dialogue. A phenomenon coined the Third Person Effect hypothesizes that people tend to believe that the influences of mass media have greater effects on others than on themselves (Mutz 1989). Trends of narcissism and superiority of feeling like one does not need media influence to form an opinion can surface under this effect through senses of independence and the strength of one's beliefs. This theory surrounding this effect can help to explain and contextualize behaviors in interaction with others, providing a theoretical base for my analysis of dialogues that focus on contentious social topics where personal belief systems in relation to others' opinions becomes the crux of the conversation. This is important because, in a world of blurred lines in the political sphere — especially between international and domestic issues - people receive their information through different sources and are aware that others receive information through different sources than they do (Mutz 1989). Creating an environment of varying perspectives on both issues and other people, the Third Person Effect theory can shed light on how people interact with each other in settings where they must consider their own perspectives relative to those of their peers.

Another theme explored within individual and social perception is the role of gender in everyday interaction. Ridgeway and Smith-Lovin (1999) suggest that gender is more of a background interaction, meaning that due to the nature of socialization, most gendered performance is subconscious. Socialized gendered performances can play into perceived power hierarchies within interaction, therefore continuing to mediate and justify cultural beliefs about the genders and their roles in society through those perceptions (Ridgeway and Smith-Lovin 1999). 
Robinson and Smith-Lovin (2001) explore the role of humor in power hierarchies, theorizing that humor can act as a means of status assertion by establishing inexplicitly who is allowed and not allowed to make jokes. They also theorize that humor within the genders differs in nature; humor between men is often differentiative while humor between women is often more collaborative. In other words, if women are socialized to act less aggressively and more collaboratively in relation to men in a patriarchal society, hierarchies of power can form subconsciously as people act in accordance with what they have been taught about their positions in society relative to others and how they should act in order to best serve their roles. Humor can be used a means of tension breaking in a group setting. When tension builds in a group, members often look for direction from some source in order to diffuse awkwardness or discomfort - this source becomes a savior-like entity who is repeatedly looked to for guidance, approval, or reverence (Bion 1961). Referring back to Robinson and Smith-Lovin (2001) humor is often monopolized by those in the group who hold higher status. Humor and input are also more positively received by higher status members - typically men (Shelly 1993). If the ability to break tension using humor lies primarily with men, then there can arise a mindset within the group that a certain man (or men) occupy this savior-like position, further recycling into alreadyexisting power dynamics within the group. However, with neutral-party facilitators guiding the conversation, I suspect that this power dynamic may not be as obvious in the dialogues I observe, since the goal of the facilitators is to bring in voices equally. The facilitators may hold a unique position of power in the eyes of the participants because they work for the center and are guiding the conversation but will not often engage in tension breaking techniques in the same ways that participants do. Rather, facilitators often use tension as a tool for exploration and reflection. 
These concepts of individual relation/perception in power hierarchies will lay a base from which I can examine gendered interaction and the roles of social factors within them. By observing things such as proxemics/body language, dialogic interruption, and dialogic deference, I can speculate the role that gender may play in settings of facilitated dialogue. This will then contribute to a larger picture of participant connection in these cross-cultural conversations that can be applied to larger-scale dialogic settings.

\section{Conversation Analysis}

Mickunas (2016) specifically dissects nonverbal or indirect communication such as body language, as well as the differences within semantics and syntax in visual language in comparison to natural (spoken) language. Literature around conversational analysis methodology of dissecting roles of gender and language in context of dialogue suggest that this approach is fruitful in its ability to link gender and discourse in conversation; much of the literature is also critical, however, of conversation analytic methodology in general due to its relative nature (Stokoe and Smithson 2001). Criticisms tend to be concerned with the subjectivity of observational data, since a person's thoughts and mechanisms behind each and every minute action cannot be realistically quantified. However, I argue that observational data is valuable in conversation analysis because much of a person's demeanor and contribution to a dialogue is subconscious to some degree. As a researcher familiar with relevant literature, there is value to identifying some of these mechanisms and putting context around them in order to better understand the workings of human interaction.

Conversation analysis exists within various mediums of communication. Charles Sanders Peirce's Sign Theory (or Semiotic) explores signification, representation, and interpretation within human interaction (Atkin 2006). Drawing from this, Mickunas (2016) discusses relations 
between visual and natural languages as well as addressing the role of individual perception in understanding information presentation methods which serves as a means of understanding one's position (more specifically, in a group) relative to someone else. Observing nonverbal communication allows us to better understand and evaluate the relative and subjective nature of visual communication. For example, men often taking up more physical space in an act recently coined as "manspreading," (Jane 2016) could communicate a certain air of confidence or entitlement relative to women's postures which tend to be more conservative and therefore may be perceived differently in comparison. Conclusions of Mickunas's (2016) analysis suggest that even though the aim of visualizing and anticipating communication is to maximize understanding, this visualization is still relative and therefore cannot be wholly represented universally; it is restricted to the community of linguistics scholars — not typically a specialization found among sociologists.

Black (2008) found that women are more likely to engage in storytelling dialogueputting context around an idea to add depth and new levels of understanding through relation and emotional bonding among group members, whereas men are more likely to engage in strategic or solution-based talk that primarily focused on one idea. Tying in with contextual and relative emotional bonding, Atkins and O’Barr (1980) analyzed court trial practice manuals in a 1980 courtroom speech study that specifically instructed lawyers to not make a woman cry because jurors are more likely to take pity on and/or forgive her. Such instructions did not exist for men being questioned, as men are typically socialized and expected to be more stoic and emotionless (Shrock 2009). These findings relate to my own ideas of collaborative and intrusive/assertive dialogic interruption where women typically interrupt in a conversation with intentions to build cohesion between speakers or add a relating component (ex. "That happened to me too") in 
contrast with men who typically interrupt with intentions to either clarify or reclaim the role of the speaker. Another way to look at these speech patterns is with labels of "direct", meaning more to-the-point, and “indirect”, meaning more contextual or vague. Atkins and O'Barr (1980) referenced Lakoff's (1975) findings that women were more likely than men to use "hedges"indirect phrases like "It seems like," "I guess," and “...kind of," "superpolite forms”__.'I'd really appreciate if..." and "empty adjectives"__charming," "sweet," in their speech (96). O'Barr and Atkins found that, in alignment with Lakoff's (1975) observations, language such as this is quickly deemed less powerful in situations relative to men's more direct speech; they call this "powerless language" (104). This idea of men and women occupying different positions of power gauged just by their speech patterns further illustrates Mutz's (1989) theories of individual perceptions of peers in relation to oneself and to others in the group.

Power within speech patterns can also influence who speaks, and in what context in relation to others in the group. The Spiral of Silence, which refers to the idea that people are more comfortable sharing their opinions when they feel their views are supported by their peers, (Mutz 1989) can deepen conversation analysis by allowing observation of topical and verbal behavior within dialogue. Essentially, the Spiral of Silence suggests that people are more likely to share in dialogue when they feel supported. Inversely, if people feel their opinions are unpopular, they are more likely to remain silent for fear of being rejected by their peers and, as a reflection, society as a whole (Mutz 1989). This can be roughly observed by noting longer periods of silence when discussing a particular topic, and when/if the silent participant decides to reenter the conversation. In instances using Socratic Inquiry facilitation, facilitators will often encourage less-vocal participants to share their thoughts or thought process in the dialogue, which can help to bring someone out of the Spiral of Silence. Sometimes, though, a participant 
may rejoin conversation if someone else shares an opinion more similar to the one they hold (in opposition to the status quo opinion of the current conversation). This can be identified by observing head nodding and small vocal affirmations (“mhmm," "yep," etc.). Shelly (1993) later supported the Spiral of Silence theory with his finding that group members who are better liked are given more opportunity to speak and their input is taken more seriously and is higher revered overall (Erickson 1978).

Relevant to my study, literature on conversation analysis explores both the roles of gender and micro-level conversation. One aspect of conversation analysis is overall group dynamic - how the group is interacting as a whole entity, and how gender might contribute to a group's dialogic structure. Understanding various elements of conversation analysis will provide me with a foundation with which to compare my observational notes of participants in dialogue.

\section{Facilitation}

Facilitation is an important part of mediation and is defined as the impartial management of meetings designed to enable participants to focus on substantive issues and goals (Susskind, McKearnen, and Thomas-Lamar 1999). Susskind et al (1999) suggest that, "practitioners skilled in mediation and consensus building are frequently found at the center of visioning, decision making, and dispute resolution processes within communities and within organizations." In the case of Socratic Inquiry facilitation, facilitators remain neutral and enter dialogues without an agenda (Paul and Binker 1990), but still encourage interaction between all participants and attempt to bring some sort of closure to a dialogue with a closing question answered by everyone.

The role of facilitation in different spaces is still being explored. Understanding the roles of facilitators as mediators of conflict and neutral parties for purposes of building connection 
between people is an important step in making informed suggestions for political spaces and governmental relations. When applied to findings regarding gendered interactions and conversational analysis, the role of facilitation in dialogue can be a powerful tool in enacting future environmental and methodological alterations in the public, political, and even private spheres.

\section{METHODOLOGY}

Since I gathered data from an existing archive, my collection method was considered secondary content analysis and therefore did not require consent from participants. This study qualified for exempt review from the West Virginia University Institutional Review Board and was approved on May $15^{\text {th }}, 2018$ (protocol number 1805120435). I gathered data from a series of twenty-five dialogues from World in Conversation (WinC), the student-run center for public diplomacy at The Pennsylvania State University in University Park, Pennsylvania. I conducted a content analysis on each dialogue in order to paint a microcosm representative of more macroapplicable situations.

Conversation analysis has been employed to study topics of gender (Lakoff 1975; Stokoe and Smithson 2001), empathy emergence in intergroup dialogue (Lamm et al 2007; Nagda et al 2009), and alliance-building (Deturk 2006). Relevant to this study, Lakoff, Stokoe and Smithson, Lamm et al, Nadga et all, and Deturk have utilized this style of analysis to examine the interactions of gender and power hierarchies in dialogue. Nagda et al (2009) posit that guided intergroup dialogue is important for cultivating empathy and building connections between participants. This study adds to this literature by incorporating analysis of facilitated dialogic guidance in instances of critical self-reflection and alliance-building. 
Stokoe and Smithson (2001) offer that imposing categories onto participants can be problematic by imposing labels on people, challenging certain aspects of my own study as I interpreted my observational notes somewhat categorically by using gender and nationality as labels through which I can begin to clarify my analysis. The difference, however, is my interlocking of objective observation and subjective interpretation—-for example, objective observations such as dialogical interruption and deference could potentially be subjectively interpreted in terms of power socialization.

As well as looking at body language of participants in dialogue, I also included properties of communication in my analysis- - I accounted for other indirect methods of communication such as proxemics (nonverbal cues within body language) to assess the role of gender in peer interaction. Mutanen's (2016) research on nonverbal communication serves as a theoretical factor to which I continue to build upon using analysis of nonverbal/indirect communication as well as dialogical interruption and deference.

World in Conversation conducts hundreds of local on-campus and global dialogues via enterprise-grade video technology every semester. Dialogues are held between Penn State students and university students from WinC's partnering countries (specifically Afghanistan, Iraq, Iran, China, Israel, Pakistan, Egypt, Qatar, and Palestine) as well as military cadets in countries across the North Atlantic Treaty Organization (NATO) alliance. Using Socratic Inquiry facilitation techniques, which employs asking open-ended questions and reflective listening, trained facilitators occupy neutral parties in dialogue with intentions to explore and expand every perspective of various social topics. World in Conversation's vision is to expose the vital role of facilitation in realms of conflict and collaboration, regardless of the nature of any specific dialogue. However, the West-East/International Relations dialogues focus specifically on 
connecting students of different cultural backgrounds with one another using contentious social topics and facilitation around opinions of such topics.

Every semester at Penn State University, various professors across campus require students to attend a dialogue at World in Conversation for class credit. Students must register online at WinC's website and attend their scheduled dialogue. I used these incoming students as my convenience sample. Each dialogue at WinC is recorded and archived for the center to review facilitation methods and generate conversation prompts. I obtained my data from the online archive that documented all recorded dialogues from the Fall 2017-Spring 2018 semesters, and randomly chose twenty-five international dialogues, each with its own unique dialogue identification. To ensure the anonymity of my subjects and the retention of sensitive subject matter, all dialogues are stored in a password-protected computer to which only I have access. Observational notes were taken in a notebook, employing a system of codes to protect the identities of all subjects. Codes used in the observational notes include: (male/female symbol)F $=$ (gender of) facilitator, $\mathrm{mX}=$ male participant, $\mathrm{fX}=$ female participant, $\mathrm{d}=$ domestic student, and $\mathrm{i}=$ international student. For example, the code " $\mathrm{mXd}$ " would indicate a domestic male student.

I specifically observed dialogues between domestic American students and international students conducted at the World in Conversation center on campus. These dialogues are coded "WE," standing for "West-East Relations" and "IN," standing for "International Relations." The nature of the dialogues is the same, but WinC changed the dialogue codes in the spring semester from "WE" to "IN," which is why the dialogue IDs are different. In the interest of proxemics observation, I took notes on verbal and nonverbal interactions between participants and facilitators in the room. This includes where each participant chose to sit, general posture (more specifically, at the beginning of the dialogue to assess initial physical space being occupied by 
participants), interruption, and deference. Because some of these visual elements were somewhat difficult to objectively observe due to video quality, I chose to leave out nonverbal elements like facial expressions and instead made note of the more blatant observations previously listed. I also took notes on conversation topics and progression of the dialogue via various conversation and connection technique from both the participants' speech (storytelling, affirmation, challenging, interruption, deference, etc.) and conversation guiding techniques from the facilitators (challenging, reflection, inquiry, etc.) Notes on these interactions and techniques were considered holistically at the closure of each video to allow the dialogues to exist as whole separate entities before comparing each one individually to the others. This process begot many layers from which my conclusions were drawn. Observations of these various dialogic occurrences will help to gauge how participants are connecting with each other and how they are forming dialogue around contentious topics with people from different cultural backgrounds as well as shed light on the role of facilitation in settings of cross-cultural diplomacy. These conclusions can then be employed in formulating policy and/or operational suggestions for governmental dialogic missions as small as local community outreach or as large as international relations.

I chose to collect data from dialogues at World in Conversation because the center trains their facilitators to utilize dialogue for purposes of intra and interpersonal exploration between participants of varying cultural backgrounds. In total, I observed approximately thirty-seven to thirty-eight hours of dialogue. Over a ninety-minute (or longer, depending on the nature of the dialogue) period, facilitators attempt to cultivate a climate of mutual trust and understanding between members of the dialogue, building rapport and extending empathy to everyone present. Because participants are encouraged to dig deeper into themselves and their perspectives, it is 
likely that typical day-to-day interactive contributors such as body language and speech patterns are not the focus of anyone's attention. Because of their organic nature, I felt it would be appropriate to include proxemics in my observational notes. Along with body language and speech patterns, I used certain contextual (after facilitators had prompted further explanation, background information, and/or emotional attachment to an idea) acknowledgments such as, "oh okay," "mhm," and "alright" as proxies for measuring empathy. This became important when assessing how facilitators helped to cultivate empathy and deeper understanding within the group, and how this affected the overall group dynamic.

This climate of openness, curiosity, and frequent participant reports of leaving the center with a broadened understanding is what makes World in Conversation an appropriate and intriguing medium for data collection. The international dialogues have a great deal of potential to formulate ideas for cross-cultural diplomacy, ambassadorship, and connection technique.

\section{RESULTS}

Spatial Patterns and Posture Dominance

Observations showed that men and women tended to group together when choosing seats, even if that meant sitting closer to a facilitator. This pattern also emerged among domestic and international students, often sitting next to each other (most likely gauged by participants' phenotypic understanding of their peers). When this pattern was broken, it was usually because of participants arriving late and having to occupy the only remaining seat. In terms of spatial occupancy, men tended to take up more physical space via their body language and positionality than women, who seemed to make an effort to take up less space in the same way. For example, men often sat with their legs apart, leaning forward or leaning far back pushing their legs further into the center of the room while draping their arms over the backs of their chairs or resting them on their legs. This phenomenon, often colloquially and playfully regarded as "manspreading," 
simply refers to men's tendency to take up considerable physical space in ways that women typically do not (Jane 2016). For example, in comparison to the men, women tended to have tightly crosses legs, or at least kept their legs together when uncrossed, and kept their hands folded in their laps for a considerable amount of time.

\section{Cutoffs and Deference}

Impressionistic inferences showed consistently that men interrupted and cut off women as they were speaking considerably more than they cut off other men. Overall, men exhibited patterns of interrupting participants more in general than women interrupted anyone, regardless of gender. When women cut each other off, they did so in a way I call collaborative interruption - meaning that women would interrupt one another to either affirm what another woman is saying, or to share her viewpoint/personal experience that aligns with the speaker's remarks. There is an aura of support and affirmation behind this type of interruption, differing from a pattern of assertive/intrusive interruption -interruption for purposes of redirection or conversational reclamation/assumption — that men more often employed. To reinforce this trend, patterns within dialogic "repairs" (efforts to mend broken/interrupted dialogue) showed that women were almost always the first to defer speaking privileges to the person who had interrupted them, whereas men rarely were at all. In some cases, men simply continued speaking over someone else, leaving no room for potential deference; this resembles "stonewalling"speaking over someone and/or refusing to collaborate, therefore causing the interrupted to close themselves off from the conversation (Gottman 2017). In the case of one dialogue, a male participant continually cut off and spoke over a female participant who simply ceased to interject after a while.

Woman: I'm not really sure [about my thoughts on what danger means to me] because I've never really- 
Man: No, yeah, but what I think they're asking is how do you perceive danger?

Personally, I don't think much about it in my everyday life.

W: Well I guess-

M: A lot of my female friends say they feel in danger all the time. Do you [women in this room] feel like that?

W: Well-

M: Like they even carry mace around all the time. Even on campus.

At this point, another woman in the dialogue answered the man's question on whether women feel in danger most of the time. The woman he continually cut off did not interject. This exchange also illustrates gendered usage of direct and indirect language. The woman speaking used hedges like "well," "I guess," and "really," while the man's speech was more direct in addressing his points.

\section{Frequency and Content of Speech}

Patterns within this study emerged showing that, although women often collaborated with one another, female international students spoke the least frequently. Women tended to speak from a more personal place (personalization and storytelling, often using "I" in their responses), even bringing solution-talk to a personal place. When women utilized humor in dialogue, it tended to align with their collaborative manner of speaking as well. Men's humor was blunter, aligning with their tendency to speak more from a place of explanation or of solution (generalization, often using "we," "people," etc. in their responses) while needing more prompting from facilitators to speak from a personal point of view. For example, one dialogue showed two women speaking directly to each other about their experiences with conflicting religions in their families. Within this exchange, one woman said, "Yeah, I guess that's what we get for questioning God," (referring playfully and jokingly to the women's shared experience of growing apart from their families due to religious differences). Both shared a laugh. In another 
religion-centered dialogue, a domestic male student interjected an exchange regarding one's personal connection with religion with, "I guess I could say God screws people over a lot," and then laughed before facilitators prompted him to say more. He followed up with, "Oh, nothing. I was just kidding." These exchanges demonstrate differences between men and women in the delivery and intention of humor as well as dialogic engagement in generalized or personal language; the women showed patterns of using humor to connect with someone and used "we," whereas men seemed to employ humor to simply get a reaction and spoke about people in general.

In alignment with this blunter role, male facilitators tended to utilize more direct language, ask pointed questions, and were more likely to stop, affirm, and redirect (essentially cut off) participants ("Hold on, I'm going to stop you quick; I want to go back to what she just said for a minute"). Contrarily, female facilitators were more likely to employ more indirect language, asking comprehensive questions and using reflections that affirmed participants ("I can see how that must have been really hard; thank you for sharing that; how do you see [that experience] affecting your views on gun ownership in the US?"). This relates back to communication styles; since men are more likely to employ solution-oriented talk and women are more likely to employ personalization, it makes sense that these styles would carry over to facilitation techniques and reflect within exploratory methods.

Another emerging trend was that both men and women claimed the role of "devil's advocate" when expressing an opinion unpopular or unstated in the room to avoid offensive conflict within the group, even though this avoidance was often mitigated by facilitators in the interest of conflict exploration. Guided partially by Mutz's (1989) theory of the Third Person Effect via self-assessment in relation to others, claiming "devil's advocate" relates back to the 
idea of gauging public perceptions of one's opinions and presenting them accordingly (which sometimes means introducing a perspective without actually claiming it). When this happened in dialogue, facilitators paused conversation to dig deeper into the "devil's advocate" opinion, allowing participants to further explore a new avenue of thought; the group would then continue conversation on the topic while incorporating the new perspective. This finding suggests the importance of Socratic Inquiry facilitation in considering minority opinions; by encouraging exploration, facilitators were able to help guide participants to a more holistic understanding of their conversation and therefore to a more critical place of thought.

Patterns of interparticipant connection emerged through avenues of idea and narrative sharing as well as aligning fears, doubts, and personal struggles. For example, many dialogues showcased an actively-growing network between participants building upon similar struggles and concerns (spanning topics like family relations, political/personal anxiety, religious experience, etc.). With the help of facilitators, interacting participants seemed to have developed a sort of social tie within the acknowledgement of shared experiences; this familiarity offered solidarity between participants of like struggle and perspective. Many participants expressed feeling relief when someone expressed a similar struggle_-"That makes me feel better," "Oh thank God [laughter]," and, "Cool, me too," were all sentiments heard throughout these conversations.

\section{Facilitation Techniques and Roles}

The role of facilitators in World in Conversation dialogues is largely exploratory, even among contention and conflict. Amidst conflict, facilitators are trained to help participants explore viewpoints, beliefs, and narratives behind contention to broaden understanding of one's 
own perspective as well as someone else's in order to critically consider an issue from as many sides as possible.

Along with this exploratory goal, WinC gives facilitators a list of prompts with which to begin dialogues. These prompts allow opportunity for a variety of perspectives to be considered, especially since many are considered controversial (i.e. prompts like: "God," "guns," "danger," "What is an opinion you could not possibly believe, and what is something someone believes that you know is wrong?" etc.). These prompts tended to breed dialogues with a great deal of personal information and contention, though not so much blatant "conflict" where participants would contort back and forth or "fight." Dialogues observed in this study did not escalate to yelling or attacking, but rather confronted conflict with language like, "I don't think I agree with that because..." When people disagreed, facilitators were able to help preemptively guide the conversation to places of perspective exploration and reflection rather than simply mediate a developing argument. This technique allowed for deeper understandings of where certain opinions came from, cultivating higher levels of empathy in the group than there would have been without such facilitation. Themes of empathy emerged through phrases like, "Oh okay," "That makes more sense now," and, "I can see why you see it that way." Because of this, groups seemed to be more open to exploring unfamiliar topical territory and were better able to critically assess different views.

One technique employed by facilitators is affirmation and redirection; this technique essentially allows facilitators to cut off a participant, affirm them, and redirect the conversation to a meaningful place if the speaker(s) is/are getting off topic. For example, one dialogue about guns showed a male participant trailing off on Second Amendment rights when the question he had been asked was about his experiences in life that had led him to his current viewpoint on an 
issue. One of the facilitators in the room stopped him mid-sentence by saying, "Sorry, let me interrupt you for a second." The facilitator then affirmed the participant to avoid completely shutting him down and redirected him to the original question - "I hear what you're saying about rights, and I can see those are really important to you. But, let's get back to what's behind your perspective. Can you tell us more about what experiences you've had that influenced your opinions on stricter background checks?" This technique was useful in making dialogues as fruitful as possible in a short ninety-minute period. It served to validate participants' ideas and experiences while also keeping the group on topic.

Facilitators would also encourage deeper thought by guiding participants through opinions with questions or further prompting; frequently used were "Say more about that," "What about that is frustrating to you," "How did you arrive at that viewpoint," and "I'd like to hear what [other participant(s)] is/are thinking." Employing active listening, facilitators also often used reflective statements to affirm, question, and/or clarify points - "It sounds like [reiteration of a point]," or "I'm hearing that [reiteration of a point]." This helped participants think more critically about something they (or someone else) had said. When presented with conflict or contention between people in a dialogue, facilitators would help dissect and mediate the disagreement by asking someone, "What are you hearing from [participant(s)]?" This technique served to bring clarity to a perspective that may have remained otherwise misunderstood or misinterpreted. After engaging with one another in this way, participants seemed to move forward in dialogue with a stronger perception of their peers' perspectives, and as a result, more informed empathic regard.

Another important finding in facilitation is how facilitators would build rapport and connection between participants. One way they did this was by acknowledging the difficulty of 
sharing certain personal information and thanking participants for sharing something. This was often done by saying things like, "That sounds difficult," or "Thank you for sharing that." This technique showed participants that their input was valued, considered, and appreciated by the facilitators and the group. Another way facilitators built connections in a group was by drawing similarities between participant perspectives, backgrounds, frustrations, and cultures. This seemed especially important when engaging quieter international students in a conversation; facilitators would often attempt to overcome looming cultural boundaries by linking personal experiences between participants; they said things like, "It sounds like both of you have experienced [issue/event/etc.]" or "It sounds like you feel similarly to [other participant(s)]." By helping participants build connections with one another, facilitators were able to begin the cultivation of empathy, which has been theorized to encourage fairer and more collaborative thought (Binmore 2001).

\section{DISCUSSION}

Themes within this study showed that men took up more physical space than women. Mutanen (2016) suggests that indirect and nonverbal mediums of communication, such as proxemics, can set a tone for interaction. This could help explain why men tended to cut people off more frequently than women in general. While men did cut off other men, women were cut off by a much larger margin. Impressionistic inference suggests that women cut people off far less frequently, and demonstrated collaborative interruption, using the interruption as a means of agreement and validation and as an opportunity to build off one another. When men interrupted someone, it was typically more disruptive in nature. When repairing these interruptions, women were almost always the first to defer speaking privileges to someone else, whereas men tended to continue speaking over someone before deferral could even be given. Relating to Mickunas's (2016) views of gender, it appears subconscious gender socialization plays into how men and 
women perceive their "place" in dialogue. In some cases, when men would continually interrupt and speak over women, it created an authoritative aura in the room, as both parties were not seen as equally active and passive. Mickunas (2016) stresses the importance of this balance to have a productive discussion where voices are heard and considered equally. Facilitators played a large role here, as they invited quieter participants into the conversation and cut off conversational manipulation to make space for others in the dialogues.

Overall, women spoke more frequently than men, with more content in moments of sharing. Women employed storytelling and personalization, sharing more personal information and even relating solution-talk to a personal place. Oppositely, men employed more generalization tactics, sharing solution-talk most often unless prompted otherwise by a facilitator. The sharing of personal information frequently acted as a bridge to other intimate interactions such as empathic acknowledgment, as discussed by Black (2008). When the dialogue reached more personal places, participants were more engaged overall and seemed more willing to empathize with perspectives different from their own. When considering how both men and women seemed to claim the role of "devil's advocate" when expressing an underrepresented opinion in the room, I consider this a microcosm of dialogue in generalpeople are likely to make this claim because of the subconscious fear of rejection by our peers. Tying back to Mutz's (1989) theory of the Third Person Effect, participants could be channeling this fear of peer rejection by distancing themselves from potential conflict, speaking of a "devil's advocate" opinion as if it only affects others and not themselves. Facilitators helped alleviate these claims to "devil's advocate" by encouraging participants to explore where viewpoints are originating and building. That way, participants were able to converse organically and honestly while thinking critically about their own perspectives relative to those of their peers. 
Socratic Inquiry facilitation methodology played an important role in leading difficult and uncomfortable conversations. By utilizing methodological techniques such as affirmation, redirection, reflection, and prompting, facilitators were able to help participants cultivate empathy for one another and critically assess contentious topics. By inviting quieter individuals into the conversation, facilitators fostered an environment of equal consideration in each dialogue. Affirming and thanking participants fostered an open and empathic culture, which proved useful in mediating contention, discomfort, and unfamiliarity in culturally diverse dialogues. I predict that conversational environments such as this would be valuable in crosscultural governmental relations due to the nature of contention and cultural/personal unfamiliarity in these larger-scale decisive meetings. Socratic Inquiry facilitation has potential to be largely beneficial in situations of decision-making because it encourages exploration of perspectives, sometimes utilizing personal narratives. In cases where this was successful, groups seemed more empathic toward one another and in turn, more open to critically and fairly assessing different ideas.

\section{Limitations}

Limitations of this study primarily regard depth of individualized analysis; due to time constraints, only twenty-five dialogues were included in the observation process. Expansion on this study would benefit from a larger sample of dialogues facilitated with Socratic Inquiry methodology and could be used to further build upon the claims made in this analysis. Ideally, this expansion would include diverse samples of people in various life stages, since this study utilizes a sample of only college students which may not be representative of a general population. Although the objective of this study is to understand a wide-scope microcosm from the dialogues for purposes of macro-level application, further research on the role of Socratic Inquiry facilitation in mediation settings would benefit from individualized analysis of each 
component touched upon in this investigation. For example, future research on the conversation analysis piece of this study would potentially benefit from timestamping and dictating each dialogue while further research on empathic response may benefit from assigning more in-depth proxies for an investigator-defined "empathy" measurement (such as in Binmore's 2001 study on why and how fairness evolves). In addition, adding a racial component of analysis may be beneficial in further understanding how culturally diverse participants interact with one another (such as in Sue's 2015 publication on how race affects racial and other contentious dialogue). Finally, this study draws from very low-stakes conversation where participants are encouraged to explore rather than make decisions. While this study does advocate for usage and trial of Socratic Inquiry methodology in contentious and collaborative settings, it must also be considered that higher-stakes conversation (such as intergovernmental communication) may lead participants to engage differently.

\section{CONCLUSION}

The purpose of this study is to explore the possibilities for improving governmental relations of varying scales using microcosms of cross-cultural dialogues facilitated with Socratic Inquiry methodology and their potential to project situational similarities into more formal political conversation.

By observing individual pieces of cross-cultural dialogue such as gender interaction/operation, conversational analysis, nonverbals/proxemics, and empathy cultivation, I can broadly understand how these factors may interact in other settings of conflict mediation such as governmental relations. By better understanding the fundamental differences in communication styles in multicultural settings, as well as identifying effective means of interpersonal connection techniques, we can apply this knowledge to virtually all communicative 
realms. This study contributes to literature regarding conversational analysis, facilitation, and cross-cultural communication by providing a deeper understanding of Socratic Inquiry facilitation's role in these dialogic microcosms. Since literature of Socratic Inquiry facilitation is scarce, this study contributes a look into how this methodology works as well as avenues for future utilization.

From personal relationships to political spaces and global public diplomacy, understanding the interaction of gender roles, conversational analysis, and the role of Socratic Inquiry facilitation in cultivating connection between culturally diverse participants is a crucial step to opening wider avenues of cross-cultural communication. Using information and findings on differences between men and women could be useful in analyzing larger-scale interaction between genders, such as in the worlds of politics, business, etc., providing a point of reference for which we can begin to unlearn damaging parts of gender socialization-perhaps an avenue for future research endeavors. In addition, understanding how facilitators help guide conversation to more empathic (an, in turn, fairer [Binmore 2001]) conflict maneuvering helps posit how this type of facilitation may affect similar settings and allow for macro-level application of such methodology between political bodies in spaces of negotiation and compromise.

By analyzing the role of Socratic Inquiry facilitation in these spaces (which can be, and often are, political), we can cultivate and project theory from these microcosms into larger realms of interpersonal communication between local, federal, and international government bodies. It is crucial in settings of political dialogue to employ empathy and explore connections between people so that the most fair and effective agreements can be realized — something many people would argue is lacking from current global politics. Dialogues from World in 
Conversation are an excellent and unique medium for understanding the role of facilitation in settings of conflict and collaboration, particularly with culturally diverse participants. Analysis of WinC's international dialogues provide unique insight to the world of cross-cultural communication and mediation-a small-scale world in the organization's halls that can be used to suggest better, more effective means of larger-scale political and governmental interactions. 


\section{REFERENCES}

Anon. n.d. "World in Conversation | Center for Public Diplomacy." World in Conversation Center for Public Diplomacy.

Atkin, Albert. 2006. "Peirces Theory of Signs.” Stanford Encyclopedia of Philosophy, Stanford University, 13 Oct. 2006, plato.stanford.edu/entries/peirce-semiotics/.

Binmore, K. 2001. "How and Why Did Fairness Norms Evolve?” Proc. British Acad. 110, 149170.

Bion, W.R. 1961. "Experiences in Groups and Other Papers.” New York: Tavistock Publications Limited. 29-58.

Black, L. 2008. "Deliberation, Storytelling, and Dialogic Moments.” School of Communication Studies, Ohio University, Athens, $\mathrm{OH}$.

Deturk, Sara. 2006. "The Power of Dialogue: Consequences of Intergroup Dialogue and Their Implications for Agency and Alliance Building.” Communication Quarterly 54(1):33-51.

Gottman, John, Carrie Cole, and Donald L. Cole. 2017. "Four Horsemen in Couple and Family Therapy." Encyclopedia of Couple and Family Therapy 1-5.

Jane, Emma A. 2016. "Dude... Stop the Spread: Antagonism, Agonism, and \#Manspreading on Social Media.” International Journal of Cultural Studies 20(5):459-75.

Lakoff, R. 1975. “Language and Women's Place.” New York: Harper \& Row 1975.

Lamm, Claus, C. Daniel Batson, and Jean Decety. 2007. “The Neural Substrate of Human Empathy: Effects of Perspective-Taking and Cognitive Appraisal.” Journal of Cognitive Neuroscience 19(1):42-58. 
Mickunas, A. 2016. "The Different Other and Dialogue." Philosophy, Communication, 1.

Mutanen, A. 2016. "Relativity of Visual Communication.” Philosophy, Communication, 24.

Mutz, D. C. 1989. “The Influence of Public Perceptions of Media Influence: Third Person Effect and the Public Expression of Opinions." International Journal of Public Opinion Research, 1 .

Nagda, Biren (Ratnesh) A., Ximena Zuniga, Patricia Gurin, and Nicholas Sorensen. 2009. "Evaluating Intergroup Dialogue: Engaging Diversity for Personal and Social Responsibility.” Diversity and Democracy 12(1).

O’Barr, W.M., Atkins, B.K., 1980. “'Women's Language' or 'Powerless Language'?” Women and Language in Literature and Society, 93-110.

O’Barr, W.M., Erickson, B., Lind, A.E., Johnson, B.C. 1978. “Speech Style and Impression Formation in a Court Setting: The Effects of 'Powerful' and 'Powerless' Speech.” Journal of Experimental Social Psychology 14(3):266-279.

Page, K. and Nowak, M. 2002. "Empathy Leads to Fairness.” Bulletin of Mathematical Biology 64(6):1101-16.

Paul, Richard; Binker, A.J. (1990). "Critical Thinking: What Every Person Needs to Survive in a Rapidly Changing World." Foundation for Critical Thinking. p. 360.

Shelly, R. 1993. "How Sentiments Organize Interaction.” Advances in Group Processes 10: 11332.

Shrock, D., Schwalbe, M. 2009. "Men, Masculinity, and Manhood Acts." Annual Review of Sociology 2009. 
Smith-Lovin, L., Ridgeway, C.L. 1999. “The Gender System and Interaction.” Annual Review of Sociology, 1999.

Smith-Lovin, L., Robinson, D. 2001. “Getting a Laugh: Gender, Status, and Humor in Task Discussions." Social Forces, September 2001.

Stokoe, E.H., Smithson, J. 2001. "Making Gender Relevant: Conversation Analysis and Gender Categories in Interaction." Discourse in Society, 12.

Sue, Derald W. 2015. "The Characteristics and Dynamics of Race Talk.” Pp. 18-33 in Race Talk and the Conspiracy of Silence: Understanding and Facilitating Difficult Dialogues on Race. Hoboken, NJ: John Wiley \& Sons, Inc.

Susskind, Lawrence, Sarah McKearnan, and Jennifer Thomas-Larmer. 1999. “The Consensus Building Handbook: A Comprehensive Guide to Reaching Agreement." London: Sage Publications. 199-207. 